\title{
Consideraciones sobre la enseñanza de la historia del arte mexicano a estudiantes extranjeros
}

Oscar Flores Flores*

\begin{abstract}
Resumen: El presente trabajo es una reflexión sobre el tema de interculturalidad y globalización, abordado desde una forma particular, mi experiencia como docente de la materia de Historia del Arte. Enseñar arte mexicano a estudiantes extranjeros conlleva el reto de superar una serie de dificultades derivadas de las diferencias culturales de los alumnos. Las ideas que aquí presento se basan principalmente en el análisis del diversos instrumentos de evaluación interna aplicados en el curso.
\end{abstract}

Palabras clave: arte, enseñanza, cultura a extranjeros, interculturalidad.

"Todo arte se origina en la mente humana, en nuestras reacciones ante el mundo más que en el mundo visible en sí, y precisamente porque todo arte es "conceptual", todas las representaciones se reconocen por su estilo."

(Gombrich, 1998: 76)

La enseñanza de la historia del arte mexicano a estudiantes extranjeros implica una serie de dificultades derivadas de las diferencias culturales de los alumnos que conforman el grupo. A simple vista, esto podría resumirse con el binomio Oriente-Occidente, no obstante sería una simplificación excesiva de una problemática mucho más compleja que implica el estudio de distintos aspectos como el idioma, la religión, la historia y el arte de cada región geográfica de la cual proceden los alumnos.

Se trata de los referentes culturales cuya importancia en el proceso de enseñanza-aprendizaje no puede ser soslayada, pues en la medida en que el profesor los tome en consideración, tendrá mayores elementos para poder transmitir adecuadamente los temas de su programa, logrando con ello una cabal comprensión por parte de los alumnos de los contenidos académicos que imparte (Flores, 2006). Asimismo, debe considerarse el papel tan significativo que tienen los referentes en este complejo proceso de globalización, en donde la interculturalidad es uno de los mayores retos que debe enfrentar el docente.

* Maestro en Historia del arte. Actualmente estudia el doctorado en la misma especialidad. Profesor de asignatura del Centro de Enseñanza para Extranjeros e investigador del Instituto de Investigaciones Estéticas. Ha coordinado eventos académicos a nivel internacional y nacional. Autor de diversos artículos relacionados con el arte novohispano. 
No obstante su importancia, creo que este tema no ha sido abordado de manera concreta en los cursos de español como segunda lengua, ni en la enseñanza de contenidos culturales a los estudiantes extranjeros en situación de inmersión. Un ejemplo de lo anterior lo tenemos en el modelo de enseñanza que se ha llevado a cabo en la Unión Europea con el objetivo principal de fomentar la vinculación cultural de todos sus países miembros: El Marco Común Europeo de Referencia para las Lenguas. Aprendizaje, Enseñanza y Evaluación [MERC] (2002).

Al igual que todas las iniciativas creadas con un sentido de integración, el MERC, surgió en un contexto político, económico y cultural afín a todos los estados que componen dicha Unión. Es decir, pese a las diferencias de desarrollo y a las obvias particularidades culturales de cada uno de ellos, en términos generales se puede afirmar que todos comparten en menor o mayor grado una identidad común, resultado de una historia, que les permite asumirse como "occidentales".

Es así que cuando se hace referencia a Occidente, sabemos que este concepto tiene una implicación histórica que trasciende al aspecto puramente geográfico, pues no sólo comprende a Europa y por extensión a América, sino que aporta una serie de valores producto de una misma tradición cultural basada en dos tópicos centrales: el mundo clásico y la religiosidad judeocristiana. Ambos temas aunados a otros aspectos locales, y por consiguiente, ajenos a estas dos tradiciones, han conformado la mentalidad de los europeos dándoles un sentido de pertenencia a un mismo ámbito cultural; de tal forma que pese a las diferencias, podría hablarse de una "identidad compartida", al grado de que algunos autores, incluso, utilizan la categoría de "civilización europea" para diferenciarla de la cultura producida en otras regiones geográficas. ${ }^{1}$

Todo ello es evidente en este modelo implementado en la Unión Europea para la enseñanza de las distintas lenguas nacionales a extranjeros, así pues, entre los objetivos de su política lingüística el MERC contribuye a lograr una mayor integración entre los distintos estados que la componen, a través de una política cultural común. Por tanto, si tomamos como punto de referencia la información contenida en el MERC, se puede deducir que es un modelo hecho en Europa para Europa, lo que necesariamente condiciona el tipo de contenidos que son abordados y la manera en que serán transmitidos.

Acorde con lo anterior, en el caso de la lengua española el Plan Curricular del Instituto Cervantes [PCIC](2006) señala que:

1 Este concepto lo encontramos desde el siglo XIX en la obra del connotado historiador francés Françoise Guizot, Historia de la civilización europea. Curso de Historia Moderna desde la caída del Imperio romano hasta la Revolución Francesa publicada en 1823, hasta autores más recientes como Delmas, (1984). 
El inventario de los referentes culturales, junto con el de Saberes y comportamientos socioculturales y el de Habilidades y actitudes interculturales, constituye una de las dimensiones del análisis de la lengua desde la perspectiva de la comunicación que está en la base del esquema conceptual de los Niveles de referencia para el español. (537)

De acuerdo con lo expresado en este mismo documento: "Estos aspectos permiten al alumno el acceso a una realidad nueva sobre una base amplia e integradora en la que se imbrican los conocimientos, las habilidades y las actitudes que conforman un modo de competencia intercultural." (537)

Aún cuando en el documento en cuestión se menciona también el interés del Instituto Cervantes en "(...) dar a conocer los rasgos característicos de los países de Hispanoamérica" (537) los aspectos a los cuales se alude se relacionan básicamente con España. En principio, no habría motivos para objetar este criterio de selección temática, ni tampoco su enfoque didáctico, el cual fue desarrollado con objetivos sumamente específicos. Sin embargo, el problema no consiste en querer adaptar el MERC a unas necesidades específicas, la dificultad surge cuando se pretende adoptar un modelo foráneo a una realidad distinta para el que fue creado.

Ello se hace evidente en el capítulo 10 del PCIC (2006) en donde se definen los referentes culturales como un tipo de "conocimiento enciclopédico" que va desde las características geográficas, políticas y económicas de los países hispanos, hasta el conocimiento de su patrimonio histórico y cultural. Éste, le proporcionaría al alumno nociones complementarias para poder comprender mejor la "identidad histórica y cultural de la comunidad a la que accede a través del aprendizaje de la lengua" (538).

Así por ejemplo en el inventario de contenidos culturales que se incluyen en el PCIC (2006: 265-266), en el apartado correspondiente a las artes plásticas se hace la siguiente propuesta: 


\begin{abstract}
CuAdro 1
Inventario de contenidos culturales del Plan Curricular del Instituto Cervantes
\end{abstract}

\begin{tabular}{|c|c|c|}
\hline Fase de aproximación & Fase de profundización & Fase de consolidación \\
\hline $\begin{array}{l}\text { Importancia y lugar } \\
\text { que ocupan las princi- } \\
\text { pales obras pictóricas } \\
\text { del mundo hispano en } \\
\text { la historia de la pintura } \\
\text { universal. }\end{array}$ & $\begin{array}{l}\text { Autores y obras repre- } \\
\text { sentativas de diferentes } \\
\text { épocas y movimientos } \\
\text { artísticos. }\end{array}$ & $\begin{array}{l}\text { Cronología de la historia } \\
\text { de la pintura española e } \\
\text { hispanoamericana. } \\
\text { Grandes movimientos y } \\
\text { tendencias: de la pintura } \\
\text { románica a las últimas } \\
\text { tendencias. }\end{array}$ \\
\hline $\begin{array}{l}\text { Las Meninas de Ve- } \\
\text { lázquez, Las señoritas } \\
\text { de Aviñon, El Guernica } \\
\text { de Picasso y los au- } \\
\text { torretratos de Frida } \\
\text { Kahlo, importancia de } \\
\text { Picasso en la pintura del } \\
\text { siglo XX. }\end{array}$ & $\begin{array}{l}\text { Retratos de monarcas } \\
\text { españoles de los siglos } \\
\text { XVI y XVII, el "arte cul- } \\
\text { to" de hispanoamérica } \\
\text { en el siglo XIX, los mu- } \\
\text { ralistas, interpretación } \\
\text { de los movimientos eu- } \\
\text { ropeos: realismo, natu- } \\
\text { ralismo, nacionalismo e } \\
\text { indigenismo, el cubismo } \\
\text { tropical. }\end{array}$ & $\begin{array}{l}\text { Pintura barroca. Valor } \\
\text { que se da a la pintura } \\
\text { barroca como reflejo } \\
\text { del esplendor de un } \\
\text { periodo histórico y } \\
\text { artístico de la historia } \\
\text { de España. }\end{array}$ \\
\hline
\end{tabular}

Como se puede apreciar, el criterio con el que se han escogido los ejemplos responde, - desde mi punto de vista-, a un enfoque eurocéntrico, en el cual las manifestaciones artísticas peninsulares han sido incluidas dada su importancia en la pintura universal, en tanto que los movimientos hispanoamericanos son vistos como pálidos reflejos del arte europeo, por lo cual sus valores son relativos, es el caso del "arte culto" del siglo XIX en Hispanoamérica o las interpretaciones exóticas de los movimientos de vanguardia, dadas las etiquetas con las que son calificados (v.g. "cubismo tropical").

Con base en lo anteriormente expuesto, podemos señalar que si los contenidos culturales hispanoamericanos son abordados tan superficialmente, no debe extrañarnos que los referentes de los estudiantes extranjeros originarios de otras áreas culturales, sean ignorados. 
Es así que en la enseñanza del español como lengua extranjera que propone el Instituto Cervantes no se consideran los referentes culturales de las lenguas de origen de los alumnos, de manera inversa lo que considera son los referentes culturales de la lengua meta, ${ }^{2}$ es decir, no se están planteando como punto de partida, sino como punto de llegada. Considero que este esquema metodológico presupone una homogeneidad cultural de todos los estudiantes a los cuales está destinado, (estudiantes procedentes del mundo occidental), pues no hay ninguna mención dirigida al docente sobre la importancia que debe poner a las particularidades culturales de cada uno de ellos, las cuales dadas sus diferencias y diversidad, deberían ser consideradas.

En el caso concreto del Centro de Eneñanza para Extranjeros (CEPE) de la Universidad Nacional Autónoma de México (UNAM), estaríamos hablando de un público estudiantil muy distinto, al que desde mi punto de vista, presupone el modelo cervantino, pues la heterogeneidad de su origen es mayor.

Por tanto considero que para una correcta enseñanza de la lengua española, el docente no debe de pensar únicamente en la enseñanza de la cultura de nuestro país, pues esto redundaría en una visión parcial y limitada de los contenidos académicos de las materias que se imparten. En este sentido el reto en el Centro debe partir de dos premisas centrales: qué tipo de alumno ingresa en el CEPE y qué tipo de alumno queremos que egrese. La primera interrogante implica elementos de muy diversa índole, como la nacionalidad, el grado de escolaridad y el nivel socioeconómico. La segunda cuestión se encuentra relacionada con el tipo de contenidos académicos, tanto lingüísticos como de las áreas de cultura (arte, historia y literatura) que deben ser incluidos y la forma en que serán enseñados.

Uno de los principales problemas que enfrenta el docente que imparte sus clases en un centro de enseñanza de español como segunda lengua, es la heterogeneidad cultural de los alumnos y la presencia de elementos extra lingüísticos, como la edad, el nivel de escolaridad de los estudiantes y los objetivos que ellos tienen para aprender el idioma español. Ambos aspectos son fundamentales ya que si no son evaluados adecuadamente pueden propiciar caer en generalizaciones vinculadas con una serie de estereotipos, que en vez de apoyar la transmisión de los contenidos académicos de las distintas asignaturas, interfieren negativamente, ya que los objetivos planteados al inicio del curso no se concretan.

Cuando inicio mi curso, uno de los primeros ejercicios que realizo en clase es la comparación entre una pintura del renacimiento italiano y una pintura china de esa misma época, el objetivo central es propiciar que el alumno las describa.

2 En lingüística aplicada, se denomina de esta manera a la lengua extranjera que una persona está aprendiendo de manera formal, encontraste con la lengua de origen o lengua materna. Vid., Richards, J.C. et al., 1997: 242. 
A este respecto, después de revisar los ejemplos relativos a distintos cursos, seleccioné los más representativos, que muestran una serie de coincidencias sobre la forma en que estudiantes procedentes de distintos contextos culturales expresaron sus opiniones sobre sendas pinturas.

Antes de consignar las opiniones de los alumnos, considero pertinente explicar las razones del por qué utilizo en este texto, el adjetivo "oriental" en vez de "asiático".

Dentro del ámbito cultural anglosajón el término "oriental" es considerado políticamente incorrecto ya que tiene una connotación despectiva, a diferencia del mundo hispánico en donde ambos términos tienen un significado diverso. El adjetivo oriental incluye principalmente, a los habitantes de China, Japón y Corea, hace referencia de manera inequívoca a una realidad geográfica muy concreta, por lo que no es casual que las frases: "Cercano Oriente", "Medio Oriente" y "Lejano Oriente" se escriban con mayúsculas, es decir como nombres propios relacionados con una geografía específica que suele contraponerse con el término "asiático" que es mucho más amplio, pues se aplica a todos los habitantes del continente.

A este respecto, si se consulta el Diccionario de la lengua española (1992: 1487) oriental es definido como: "perteneciente o relativo al Oriente" y a su vez Oriente se define como: "Asia y las regiones inmediatas a ella de Europa y África". (1487) Es decir si retomáramos de manera literal este concepto podría parecer que la utilización que yo hago del término es incorrecta, no obstante, debe recordarse que así como una definición suele tener diversas acepciones, de acuerdo con su significado lingüístico, también es cierto que hay valores distintos asociados a la lengua y otros de tipo cultural que no siempre se han incorporado al diccionario, lo que no implica que no existan.

Acorde con ello, no sólo hago referencia a la forma en que el adjetivo es utilizado por los hispanohablantes, pues basta recordar que aún cuando un considerable número de europeos estarían vinculados con esa definición, con toda seguridad puede afirmarse que un ruso o un griego se identificarían a sí mismos como "orientales". Para reforzar esta idea basta recordar que se habla más de una Europa del este que de una Europa oriental. De manera análoga, en la mentalidad occidental difícilmente se puede asociar a un habitante de Irak, de la India o de Afganistán como "orientales".

Desde mi punto de vista, la vasta heterogeneidad cultural de Asia impide que para un occidental el término asiático pueda utilizarse de manera indistinta para referirse a sus diversos habitantes. Por otra parte, remito también a mi experiencia personal como estudiante extranjero en una situación de inmersión. De 1988 a 1990 tuve la oportunidad de estudiar en la antigua Unión Soviética y relacionarme con estudiantes de distintos países de dicho continente. Durante ese tiempo me pude percatar que debido a las profundas diferencias culturales que ellos tenían entre sí 
(religión, idioma, costumbres y prejuicios raciales), los estudiantes procedentes de Asia no reconocían ningún tipo de vínculo con aquellos compañeros que no eran originarios de regiones culturalmente afines. A diferencia de estos, aun cuando entre ellos también existiera una gran diversidad lingüistica y cultural, siempre se asumían como tales. Por todo ello, concluyo que la utilización del adjetivo "oriental" es de índole cultural, mientras que el de "asiático" es meramente geográfico.

En el caso concreto de los estudiantes del CEPE, sucede algo semejante, cuando existe un grupo multicultural conformado por estudiantes de Europa y Norteamérica en quienes se puede detectar cierta afinidad por la coincidencia de valores culturales que ellos comparten. A ello habría que agregar otros dos aspectos de tipo académico que tienen que ver con la enseñanza del español como segunda lengua y la enseñanza de contenidos culturales a estudiantes no hispanohablantes.

En el primer caso se trata de la relativa facilidad que tienen los alumnos occidentales para aprender un idioma de su misma familia lingüística. Con ello no me refiero al parentesco que puede haber únicamente entre dos o más lenguas del mismo grupo, que en el caso europeo, grosso modo serían, romances, germánicas y eslavas, sino al hecho de que salvo las lenguas fino-ugrias, todas ellas forman parte de la familia indoeuropea. ${ }^{3}$ En cambio, los estudiantes de Asia cuyos idiomas no pertenecen a esta familia, no desarrollan con la misma facilidad las habilidades lingüísticas para aprender un idioma extranjero diferente al propio, en este caso el español, lo cual se ve reflejado en la forma que ellos tienen para aprenderlo en comparación con sus compañeros occidentales. Lo anterior es evidente en la velocidad con la que asimilan las cuestiones gramaticales, la capacidad para comprender tanto la lengua hablada como la escrita y su disposición para reproducir los valores fonéticos adecuadamente.

Con relación al segundo punto, los estudiantes europeos y norte-americanos comparten los mismos referentes culturales, en contraposición con los asiáticos, quienes al carecer de ellos, asimilan con mayor dificultad los contenidos de la clase, tanto aquellos que son estrictamente lingüísticos y que están orientados a propiciar el desarrollo de las distintas competencias y habilidades, como los relacionados con los conocimientos académicos propios del arte, la historia y la literatura.

Otro aspecto en donde los referentes ocupan un lugar importante es en el desarrollo de los saberes y comportamientos socioculturales y en el de habilidades y actitudes interculturales, que son mencionados en el curriculum del Instituto Cervantes, pues en la medida en que a un alumno extranjero dichos saberes y comportamientos le sean familiares, podrá asimilarlos y aplicarlos más rápidamente.

3 Para un panorama general sobre las características de esta familia lingüística. Vid., Villar, F. (1971); Benveniste, É. (1983) y Bosch P. (1987). 
Como se verá a continuación, esta carencia de referentes culturales es decisiva al momento en el cual el alumno tiene que analizar una obra de arte en particular, como es el caso de una pintura, cuyas características formales responden a una serie de elementos propios de un proceso cultural específico y de un contexto histórico concreto.

\section{CUADRO 2}

Opiniones de estudiantes occidentales y orientales

\begin{tabular}{|c|c|}
\hline $\begin{array}{l}\text { Rafael Sanzio } \\
\text { La virgen del Prado, } \\
\text { (ca. } 1504 \text { - 1505), } \\
\text { Kunsthistorisches Museum, } \\
\text { Viena, Austria }\end{array}$ & $\begin{array}{l}\text { Kao K'o - Kung } \\
\text { Paisaje después de la lluvia, } \\
\text { (ca. 1300), } \\
\text { Museo del Palacio Nacional } \\
\text { de Taipei }\end{array}$ \\
\hline $\begin{array}{l}\text { "En la imagen de Italia, vemos un } \\
\text { mundo donde el humano está al } \\
\text { centro; la naturaleza lo rodea... el } \\
\text { pintor embellezó todo para mostrar } \\
\text { que es el trabajo de Dios." }\end{array}$ & $\begin{array}{l}\text { "La imagen de China es muy sen- } \\
\text { cilla y natural pero no es muy real... } \\
\text { se debe a la ausencia de cristianismo } \\
\text { en el país." } \\
\text { (Christoper, Estados Unidos) }\end{array}$ \\
\hline $\begin{array}{l}\text { "Pintada en la Italia del Renacimiento } \\
\text { tiene un gran realismo y está repre- } \\
\text { sentado los personajes bíblicos en } \\
\text { la forma muy humana, corpalar que } \\
\text { tienen un ideal de belleza del XIV } \\
\text { siglo." }\end{array}$ & $\begin{array}{l}\text { "En esta imagen de China ya no al- } \\
\text { canzamos ver las figuras humanas que } \\
\text { nos puede indicar que su importancia } \\
\text { no fuera mayor que esa de la natura- } \\
\text { leza. Es también menos realista." } \\
\text { (Agatha, Polonia) }\end{array}$ \\
\hline $\begin{array}{l}\text { "En esta pintura nosotros podemos } \\
\text { encontrar un paisaje naturalista y } \\
\text { realista." }\end{array}$ & $\begin{array}{r}\text { "En esta pintura podemos encontrar } \\
\text { un paisaje naturalista pero abstracto." } \\
\text { (Zhang Ling, China) }\end{array}$ \\
\hline $\begin{array}{l}\text { "En la pintura de Rafaelo se ve la } \\
\text { perspectiva, hay la profundidad y } \\
\text { muy realismo." }\end{array}$ & $\begin{array}{l}\text { "El paisaje del dibujo chino repre- } \\
\text { senta la naturaleza ideal. Aquí la na- } \\
\text { turaleza es más importante que los } \\
\text { hombres." (Kang Hye Won, Corea) }\end{array}$ \\
\hline
\end{tabular}


CUAdRo 2

(continuación)

\begin{tabular}{|c|c|}
\hline $\begin{array}{l}\text { Rafael Sanzio } \\
\text { La virgen del Prado, } \\
\text { (ca. } 1504 \text { - 1505), } \\
\text { Kunsthistorisches Museum, } \\
\text { Viena, Austria }\end{array}$ & $\begin{array}{l}\text { Kao K'o - Kung } \\
\text { Paisaje después de la lluvia, } \\
\text { (ca. 1300), } \\
\text { Museo del Palacio Nacional } \\
\text { de Taipei }\end{array}$ \\
\hline $\begin{array}{l}\text { "En la pintura occidental, paisajes } \\
\text { son menos importantes que los hom- } \\
\text { bres, por eso tres figuras en la pintura } \\
\text { ha sido representado más significa y } \\
\text { grande. En el mundo occidental se } \\
\text { consideraba que el más importante } \\
\text { es humano... por eso ellos empe- } \\
\text { zaron a destruir naturaleza para los } \\
\text { humanos viven mejor." }\end{array}$ & $\begin{array}{l}\text { "En esta pintura oriental el artista } \\
\text { sólo dibujo las montañas y árboles. } \\
\text { Hay ninguna consideración para } \\
\text { representar los hombres. Muestra los } \\
\text { sentimientos a través de los paisajes. } \\
\text { En mundo oriental, se consideraba } \\
\text { que la importancia de todos los vi- } \\
\text { vos es igual, por eso trataba la natu- } \\
\text { raleza con respeto." } \\
\text { (Maki Nogaito, Japón) }\end{array}$ \\
\hline
\end{tabular}

Como se puede apreciar en el cuadro 2, la descripción de ambas pinturas, no solo refleja un conocimiento de tipo pictórico aprendido en el curso, también muestra una forma absolutamente distinta de ver el mundo. ${ }^{4}$

En efecto, mientras los alumnos occidentales describen las dos obras desde un punto de vista narrativo y aún cuando su atención no se ocupa en identificar un tema concreto, los elementos centrales de su análisis son los personajes y la manera en que son representados. El en caso de los orientales su atención se centró en la descripción del paisaje, resaltando el aspecto simbólico que tienen los elementos incluidos en la composición.

Por tanto, es evidente que el acercamiento que hacen los dos grupos de estudiantes a dichas pinturas, están condicionados por sus respectivos referentes culturales de origen, es así que "siendo los actos de ver y pensar, inseparables, debemos admitir que cada descripción ya contiene alguna interpretación..." (Pächt, 1989: 56), esto es claro en los dos alumnos occidentales, pese a sus diferencias (formación protestante el primero y católica la segunda) provienen de una tradición permeada por el cristianismo, en donde el hombre es el centro del Universo, por lo que su descripción obedece a los mismos esquemas.

4 Para llevar a cabo esta selección, se tomaron en cuenta alrededor de 30 exámenes realizados por estudiantes procedentes de Europa, Estados Unidos y Canadá, así como del Lejano Oriente e inscritos en el curso intensivo "La pintura Virreinal en la Nueva España" durante los años de 2002 a 2008. 
De manera análoga, aunque en sentido inverso, los estudiantes orientales poseen también un sustrato religioso y filosófico común en donde las creencias budistas, confusionistas y shintoistas comparten una visión del mundo, ya que se afirma que el hombre está en la misma escala de valores que tienen los animales, las plantas y las montañas. En este sentido, se puede decir que "el vocabulario relativamente rígido de la tradición china opera como una pantalla selectiva que sólo admite los rasgos para los que dispone de esquemas." (Gombrich, 1998: 73)

Otro ejemplo semejante que refleja esta misma problemática lo tenemos en la descripción de la pintura de la Nueva España, pues ante la ausencia de referentes de tipo religioso los estudiantes orientales utilizan diversos recursos para poder subsanar sus "carencias" y poder cumplir con las tareas asignadas. En este sentido "Diego", cuyo verdadero nombre es Jun Dai Suk de Corea, al describir una pintura del artista barroco Cristóbal de Villalpando cuyo tema es La Santísima Trinidad, lo hizo de la siguiente manera:

"Dios, Jesús y Espiritud es tringulidad, es decir, Dios es Jesus, Jesus es espiritud, Espiritud es Dios. Dios viene en el suelo por hombre para salvar todo el mundo, pero cuando tenía 33 años fue muerto por los ejércitos romanos que no les gusta Dios, tres días después de crucificar, revive y todo el mundo se asusta por su renacimiento."

Es evidente que el alumno no entiende el dogma representado (lo cual es muy comprensible), pero si refleja una relativa asimilación de la explicación que escuchó en el salón de clase, tanto en el aspecto iconográfico como en el histórico, como se aprecia en su breve reseña del tema de la Pasión de Cristo.

Un caso por demás ilustrativo de este mismo problema de ausencia de referentes es el de "Elena", estudiante coreana cuyo nombre es Park Bo Kyung, quien realizó su trabajo, a partir de una descripción de una obra perteneciente a la colección del Museo Nacional de Arte de la ciudad de México, se trata de Santa Úrsula del pintor Hipólito de Rioja:

"Voy a describir la pintura Hipólito de Rioja. Según el diccionario, dice que Hipólito es hijo de Teseo en mitología griega. El fue matado por el dios del mar y la palabra Rioja es un lugar de España, pero no creo que este título tenga relación con la pintura. Arriba de la pintura hay una señora abrazando un niño alrededor de la señora hay

5 Cabe mencionar que una práctica común entre los estudiantes coreanos que asisten al CEPE, es adoptar un nombre occidental de su preferencia; de acuerdo con sus propias palabras, con esta acción su proceso de incorporación a la sociedad en la que están viviendo será mucho más sencilla. 
muchos ángeles. La luz está brillándolos, pienso que eso significa la paz, porque debajo de la señora se aparecen los aspectos de la guerra. Los soldados están matando las mujeres. La derecha de la pintura hay un barco el que la luz está brillando, me parece que ese barco simboliza los conquistadores. En el centro de la guerra hay una señora quien está orando mientras está herida de una flecha. Por todos esos me imagino que la pintura simboliza la paz."

Al analizar el lienzo, nuestra alumna se percató que la información procedente del diccionario que consultó para elaborar su trabajo de curso, no correspondía en absoluto con el tema representado en la obra que eligió para su estudio. No obstante, es significativo que Park realizara un esfuerzo notable por explicar una pintura cuya temática estaba muy lejos de ser identificada correctamente. En realidad se trata de una confusión derivada de un "error de lectura" ya que la alumna confundió el nombre del pintor (Hipólito de Rioja) con el título de la obra Santa Úrsula y las once mil vírgenes una historia de raigambre medieval, desconocida, incluso, por muchos cristianos. Al anterior problema iconográfico, habría que agregar aspectos puramente formales, como la compleja composición y el manejo de luces y sombras. Ante la dificultad de describir la pintura con criterios puramente plásticos, "Elena" procedió a la libre interpretación confiriéndole a la luz el significado de la paz.

De manera semejante lo hace "Sofía" (Park Eun Youg), otra alumna coreana, quien antes de describir la pintura nos advierte:

"No tuve una religión, no sé sobre la Biblia y el origen de Cristo. Solamente estoy aquí en México. A veces iba a la iglesia con mi casera."

Pero haciendo un esfuerzo de interpretación, al analizar la pintura El Sagrado Corazón de María del Museo Nacional de Arte, de la ciudad de México, describe lo siguiente:

"El corazón que está situada en el centro quizo expresar Jesús... la paloma simboliza la paz... el corazón tuvo dolor de físico pero no tuvo dolor de espíritu."

De esta manera se puede ver cómo para dos estudiantes del mismo país, elementos plásticos muy diversos pueden otorgarle un mismo significado, así pues si para "Elena" la luz simbolizaba la paz, para "Sofía" este valor correspondía a la paloma, quizá esto último se explique por una posible interferencia de tipo occidental que todos nosotros conocemos, es decir, la paloma como símbolo de la paz y armonía (Chevalier y Gheerbrant, 1991: 796-797). 
Muy diferente a los ejemplos de los estudiantes orientales que he comentado, es el caso de los europeos cuyos trabajos necesariamente implican un análisis diferente, dado el conocimiento e inquietudes que presentan. Así pues, Jasminka Magilec, alumna croata del nivel Intermedio 3, inició su trabajo con las siguientes palabras:

"Para empezar necesito admitir que me costó mucho trabajo hacer este análisis, porque no sólo, no tengo el suficiente conocimiento sobre el tema del cuadro o sobre el simbolismo de los objetos pintados, sino que tampoco puedo estar segura al $100 \%$, de que estilo artístico es la pintura. Pienso que me faltan cursos anteriores que no escuché y para pasar este lapso investigué mucho en internet. Algunas informaciones sobre el pintor, el tema y el momento histórico tienen referencia a clases que escuché y los informaciones que encontré en internet, pero el análisis del cuadro es mío y por eso pude tener muchos errores que se basan en mi poco conocimiento. Eso quiero estacar para no hacer el error sobre el que habla el señor Gombrich: 'Es infinitamente mejor no saber nada acerca del arte, que poseer esa especie de conocimiento a medias propio del esnob'".

El comentario, además de mostrar otro aspecto importante del mundo globalizado, en el cual la consulta de información en la red es inherente a los hábitos de estudio de nuestros alumnos, nos remite también a las lecturas que se recomiendan en clase. En este caso, Jasminka basa su comentario en las palabras de Gombrich (2007: 36), expresadas en la introducción de su Historia del arte quizá el mejor libro de divulgación sobre esta disciplina y uno de los más difundidos a nivel internacional. En su obra el autor lejos de dar una nómina interminable de nombres, fechas y estilos, pone especial énfasis en la manera en la cual debe apreciarse el "arte", para ello exhorta al lector a ver las obras con una actitud abierta, libre de prejuicios y pretensiones. Acorde con ello, la alumna se apoyó en las palabras de este connotado especialista, con la convicción de que su comentario, aún cuando no fuera del todo acertado, si sería auténtico.

Más adelante, Jasminka procedió a describir el cuadro en cuestión con los siguientes términos:

"El cuadro representa una escena del Antiguo Testamento, típica para la época, con una composición básicamente simple pero teatral... La escena es muy sencilla y la composición es simétrica y limpia... El artista ha dividido la composición en dos secciones, el 
primer sección representa la Judith con colores más obscuros y su imagen es más real, y el segunda sección que es la escena detrás de la Judith donde dominan las colores pasteles, pero la imagen es poco diluida y sirve solamente para estacar más personaje de Judith... Ella está pintada como una reina con su corona de oro, puesta dentro de un aura sagrada, como una representación de su santidad, pero su personaje más representa una mujer bella de su época, que una santa, como que su santidad está perdiendo frente a su humanidad... A mí me gusta esta obra porque es poquito diferente de otras obras religiosas de Villalpando y de esta época. El cuadro no está tan obscuro, no tiene tan grandes contrastes. Los colores son brillantes y su mensaje es claro y fuerte."

Huelga decir la riqueza de este comentario, pues es evidente que la alumna hizo un notable esfuerzo por analizar la pintura, de acuerdo con los parámetros establecidos en clase, es decir, estudiar la obra de arte desde un punto de vista formal. Con base en este criterio, debía privilegiarse el análisis estilístico, la composición, el empleo del color y el manejo de las luces y las sombras, todo ello con el objetivo de brindar al alumno las herramientas para poder comprender e interpretar las artes plásticas, sin necesidad de depender sólo de la iconografía, que por su misma naturaleza puede ser menos atractiva y en muchos casos muy difícil de entender, sobre todo si se trata de los estudiantes de los países orientales. Sobre este punto sólo queda decir que aún cuando Jasminka (cuya carrera universitaria estaba relacionada con las finanzas), reconoció su falta de conocimientos en muchos aspectos relacionados con la obra de arte, el estudio tan acertado de la pintura que analizó, fue posible gracias a los referentes culturales de occidente.

Finalmente, voy a consignar un ejemplo en donde lejos de describir o analizar el cuadro de su elección, Sandra McCann estudiante británica, reflexiona en torno a un concepto fundamental de la historia del arte. Para poder explicar la pintura La Virgen y el Niño con San Francisco de Borja y San Juan de Regis del pintor novohispano Miguel Cabrera, Sandra decidió más que hacer un análisis formal del cuadro abordar otros aspectos, entre los que destaca el siguiente:

"Cuando pienso sobre arte de los siglo XVI a XVIII, una palabra en particular está en mi mente: "influencia". La influencia es un tema repetido por todas partes en el mundo del arte. Hay muchos tipos de influencia en el arte. Tres temas de influencia más importantes para los siglos son la influencia de la iglesia a España, la influencia de España en las personas indígenas y la influencia 
de los descubrimientos científicos en el arte en general. Es fácil entender que la evolución del arte y la evolución de la historia están unidas... Cuando examinamos la historia y las pinturas de estos siglos, podemos ver que los dos tenían influencia del otro. Podemos aprender sobre las vidas y la historia de la gente de esta época de las pinturas. Cuando la vida de la gente estaba cambiando, las pinturas estaban cambiando también. Sin embargo, podemos ver la influencia del pasado en todos los siglos, y hoy también."

La singularidad de este comentario frente a los ejemplos anteriores, radica en la forma en la que Sandra inició su reflexión, ya que la historia representada y los valores formales de la obra son relegados en virtud de un concepto más significativo. Efectivamente, para ella fue más importante el análisis de un problema concreto de la historia del arte: el concepto de "influencia", por medio del cual, temas como la transmisión de modelos, la circulación de obras y artistas y los orígenes de un estilo, adquieren mayor importancia en la comprensión de una manifestación plástica. Es así que en este ejemplo concreto, la alumna partió de una reflexión más profunda, para poder analizar su pintura. Ello refleja una posible formación humanística y una notable capacidad de comprender la complejidad del proceso creativo de una obra de arte. Asimismo, expresa también una voluntad de explicar el fenómeno artístico como un hecho histórico y la facilidad de establecer relaciones entre los distintos aspectos del arte.

Es así que a partir del estudio de los ejemplos enunciados podemos inferir que la diversidad de respuestas radica en los distintos niveles de lectura que puede tener una obra de arte. Estas discrepancias pueden estar relacionadas con el carácter multicultural de los grupos a los que he impartido clases a lo largo de estos años, por lo que nuevamente vuelvo al tema con el que inicié este ensayo: la necesidad de conocer los referentes culturales que tienen nuestros alumnos, pues es un requerimiento esencial en esta época de globalización en donde la interculturalidad es una meta todavía difícil de alcanzar.

En el caso concreto del CEPE, considero que los cursos de cultura pueden organizarse a partir de las respuestas que se obtengan de las siguientes preguntas: ¿Qué nivel de español tienen los alumnos?, ¿cuál es su perfil académico?, ¿qué referentes culturales tienen?, ¿cuáles serían los temas de estudio que podrían interesarles?, ¿cuáles son nuestras expectativas como profesores de historia del arte mexicano a estudiantes extranjeros no hispanohablantes?

Una adecuada respuesta a estas interrogantes, podría aportar mayores elementos para la programación de los cursos; el diseño de materiales didácticos que utilizamos en el salón de clases; la elaboración de manuales especializados o la enseñanza a través de medios electrónicos a distancia; todo ello desde una perspectiva intercultural y multidisciplinaria. 
Así pues, través de una metodología teórico-práctica que incluya conocimientos de historia del arte y aquellos vinculados con la enseñanza del español como lengua extranjera, el profesor brindará al alumno una aproximación a los temas de estudio planteados en su programa de curso. Con ello, no sólo transmitirá adecuadamente al alumno los contenidos académicos propios de su especialidad, sino que de manera indirecta contribuirá también a desarrollar las competencias y habilidades lingüísticas de sus estudiantes.

Para concluir, quiero enfatizar el aspecto más subjetivo, pero al mismo tiempo el más característico de la apreciación artística que buscan nuestros estudiantes: el goce estético; un tópico recurrente entre los individuos que quieren relacionarse con nuestra área de estudio. Por ello, finalizo esta reflexión con las palabras de la alumna ucraniana Oksana Kozhik, quien después de escribir su comentario sobre una obra del pintor Rafael Ximeno y Planes, expresó:

"A mí me gusta esta imagen porque cuando observe la pintura, yo siento paz, tranquilidad e ilumina mi vida." 
Bibliografía

Benveniste, É. (1983) Vocabulario de las instituciones indoeuropeas. Madrid: Taurus. Bosch, G. (1987) El problema indoeuropeo. México: Fondo de Cultura Económica. CELIS, Á. y Heredia, J. [coords] (1987) Lengua y cultura en la enseñanza del español a extranjeros. Actas del VII Congreso de ASELE. Cuenca: Universidad de Castilla La Mancha.

Chevalier, J. y Gheerbrant, A. (1991) Diccionario de los símbolos. Barcelona: Herder. Delmas, P. (1984) Historia de la civilización europea. México: Fondo de Cultura Económica.

FLORES, O. (2006) La importancia de los referentes culturales en la enseñanza de la pintura virreinal a estudiantes extranjeros. Decires, 9, (9), pp.35-48.

Gombrich, E. (1998) La verdad y el estereotipo en Arte e ilusión. Estudio sobre la psicología de la representación pictórica. Madrid: Debate. , (2007) La historia del arte. China: Phaidon.

Marco Común de Referencia para las Lenguas: Aprendizaje, Enseñanza, Evaluación, (2002). Madrid: Instituto Cervantes, Ministerio de Educación, Cultura y Deporte.

РÄтCH, O. (1989) Historia del arte y metodología. Madrid: Alianza.

Plan Curricular del Instituto Cervantes. Niveles de referencia para el español. (2006) Madrid: Instituto Cervantes (Biblioteca Nueva).

RAHLL, D. (1989) Por una ampliación del concepto de enfoque comunicativo en Estudios de Lingüística Aplicada. México: Universidad Nacional Autónoma de México, Centro de Enseñanza de Lenguas Extranjeras.

ReAl Academia Española, (1992), Diccionario de la lengua española (21 ed.) Madrid: Espasa-Calpe.

RICHARDS, J., et al. (1997), Diccionario de lingüística aplicada y enseñanza de lenguas. Barcelona: Ariel.

VILLAR, F. (1971) Lenguas y pueblos indoeuropeos. Madrid: Istmo. (Colección Fundamentos, 14). 